Journal of

Epilepsy and

Clinical

Neurophysiology

J Epilepsy Clin Neurophysiol 2006; 12 (1):25-30

\title{
Ressonância Magnética Funcional de Memória: Onde Estamos e Onde Podemos Chegar
}

\author{
Daniel Branco*,**, Jaderson Costa da Costa** \\ Serviço de Neurologia, Hospital São Lucas, PUCRS, Porto Alegre, RS
}

\begin{abstract}
RESUMO
Introdução: Ressonância functional (fMRI) é uma técnica capaz de substituir o teste de Wada como teste padrão de lateralização de memória. Atualmente, a fMRI já pode ser utilizada para estimar a lateralização de memória nos lobos temporais mediais, mas algumas variáveis devem ser tratadas de forma a garantir uma melhor qualidade dos resultados, como a intensidade do campo magnético e o paradigma utilizado. Objetivos: Neste artigo, nós revisamos os últimos avanços em neuroimagem functional de memória e buscamos esclarecer o futuro papel da fMRI no planejamento pré-operatório na cirurgia da epilepsia. Conclusões: Atualmente, paradigmas em bloco ainda são preferíveis (em vez de paradigmas relacionados a eventos e outras abordagens) e os pacientes devem ser comparados a um grupo controle, de forma que os índices de lateralização estimados possam ser clinicamente úteis. A fMRI de memória evoluirá pelo uso de campos magnéticos de alta intensidade (3T) e pelo uso de novas abordagens matemáticas para cálculo dos índices de lateralização. No futuro, ela será combinada com outras técnicas de mapeamento functional, tais como a tratografia, o EEG intracraniano, e a magnetoencefalogragia, para produzir mapas multi-modalidade. Em vez de simples figuras, no futuro, os mapas de memória serão apresentados mais provavelmente na forma de filmes funcionais.
\end{abstract}

Unitermos: epilepsia, memória, ressonância funcional, cirurgia da epilepsia.

\begin{abstract}
Functional MRI of memory: where we are and where we can reach

Introduction: Functional MRI (fMRI) is a promising technique for replacing the Wada test as the standard examination of memory lateralization. Currently fMRI can already be used to estimate lateralization of memory in the medial temporal lobes, but some variables should be treated to guarantee good-quality results, such as magnetic-field strength and design types. Objective: In this article, we review the latest advances in functional neuroimaging of memory and attempt to elucidate the future role of functional MRI as a preoperative tool in epilepsy surgery. Conclusions: At this point, blocked design still is preferable (over event-related and other approaches) and patients need to be compared to a control group, so the laterality indices may be more clinically meaningful. Functional MRI of memory will evolve by the use of high-strength field magnets (3T) and novel mathematical techniques for estimating laterality indices. In the future, it will be combined with other functional mapping techniques, such as tractography, intracranial EEG, and magnetoencephalography, to produce multi-modality maps. Rather than a picture, future memory maps will most likely be presented as functional movies.
\end{abstract}

Key words: epilepsy, memory, fMRI, epilepsy surgery.

\footnotetext{
* Golby Lab (Surgical Brain Mapping Laboratory), Department of Neurosurgery, Brigham and Women's Hospital, Harvard Medical School, Boston, USA.

** Programa de Pós-Graduação em Medicina e Ciências da Saúde, Faculdade de Medicina, Serviço de Neurologia, Hospital São Lucas, e Instituto de Pesquisas Biomédicas, Pontifícia Universidade Católica do Rio Grande do Sul (PUCRS), Porto Alegre, Brasil.

Received Jan. 16, 2006; accepted Mar. 10, 2006.
} 


\section{INTRODUÇÃO}

Os lobos temporais mediais são essenciais para processos de codificação e recuperação de memória declarativa $^{(1-4)}$. Como o LTM é um local freqüente de foco epiléptico refratário ao tratamento medicamentoso, determinados pacientes podem se beneficiar de ressecção cirúrgica do LTM afetado. Entretanto, é essencial que se entenda previamente a uma cirurgia as contribuições relativas de cada um dos dois LTMs (particularmente a contribuição do LTM contra-lateral) com relação à função de memória para evitar déficit pós-cirúrgico. Por isso, testagem de memória é realizada de rotina na avaliação pré-operatória em centros de referência para cirurgia da epilepsia. Medidas de lateralização de memória podem ajudar na avaliação da reserva funcional em ambos os lados e na definição quanto à indicação ou não de cirurgia e até que ponto estender a ressecção(5).

Tradicionalmente, o Teste do Amital Sódico (TAS ou Teste de Wada) tem sido empregado para o estudo da lateralização de memória ${ }^{(6,7)}$. O TAS consiste na injeção de amobarbital sódico, um anestésico, na artéria carótida interna, causando uma desativação temporária do hemisfério cerebral ipsilateral. Durante o período de inatividade hemisférica, é realizada testagem neuropsicológica para determinar a função cognitiva sustentada pelo hemisfério contra-lateral. O TAS foi desenvolvido inicialmente para determinar lateralização de linguagem, mas também tem sido comumente utilizado para lateralização de memória. Como um teste de memória, no entanto, o TAS sofre diversas ressalvas: tem resolução espacial e temporal pobre; ainda não está claro se o seu efeito é causado por desativação direta ou por deaferentação de estruturas do LTM (particularmente as regiões posteriores, supridas pela circulação posterior) ${ }^{(8)}$; não pode ser prontamente repeti$\mathrm{do}^{(9)}$; é invasivo (acarretando em riscos relacionados à cateterização $\left.^{(10)}\right)$. Mesmo assim, porque o TAS simula os efeitos da ablação cirúrgica, e em função da experiência de diversos centros com o exame, ele é considerado padrão-ouro para avaliação pré-operatória de lateralização de memória(5).

Mais recentemente, técnicas de neuroimagem funcional, como a Tomografia por Emissão de Pósitrons (PET) e a ressonância magnética funcional (RMF), têm sido avaliadas como potenciais substitutos ao TAS(5). A RMF é particularmente promissora para o estudo da memória por ser não-invasiva, ter muito boa resolução espacial, ser facilmente repetida, e permitir o estudo de múltiplas funções cerebrais. Sua capacidade de identificar ativações nos LTMs durante memorização já foi bem demonstrada em sujeitos normais $^{(11-19)}$ e em pacientes com epilepsia ${ }^{(20-27)}$.

A lateralização da memória, no entanto, é mais complexa do que a lateralização de linguagem, pois varia de acordo com o tipo de material memorizado ${ }^{(11,13,20)}$, com a estratégia de memorização ${ }^{(28-30)}$, e mesmo com o estágio de processamento de memória estudado (memorização X reconhecimento, por exemplo). Tradicionalmente, em estudos de lesões cirúrgicas, o LTM esquerdo tem sido mais relacionado com a memorização de estímulos verbais ${ }^{(31,32)}$, enquanto que o LTM direito tem sido mais relacionado com a memorização de estímulos não verbais ou visuais $^{(33,34)}$. Mais recentemente, essa lateralização de memória relacionada a material foi também observada por estudos de RMF nos lobos frontais ${ }^{(14,35)}$ e nos LTMs $^{(11,13,20,36)}$. No entanto, em nenhum desses estudos, a dicotomia de memória verbal à esquerda e memória visual à direita foi tão claramente estabelecida quanto a lateralização de linguagem para a esquerda. De fato, esses estudos demonstram que existe uma "tendência" ou "preferência" de lateralização conforme o tipo de material, onde, na prática, ambos os LTMs estão continuamente envolvidos em maior ou menor grau.

Uma outra questão relevante à lateralização de memória nos LTMs é o paradigma utilizado (em bloco ou relacionado a eventos). Os paradigmas em bloco são os mais poderosos, identificando mais consistentemente atividade nos LTMs. No entanto, como as categorias de estímulo em cada bloco precisam ser definidas previamente ao estudo, não é possível categorizar posteriormente estímulos que foram memorizados com sucesso e estímulos esquecidos, já que apenas após o estudo se pode saber quais estímulos se enquadram em cada uma dessas categorias. $\mathrm{O}$ paradigma relacionado a eventos possui a vantagem de permitir que a categoria de cada estímulo seja definida a posteriori, o que é principalmente utilizado para avaliar o efeito da memorização bem sucedida. Paradigmas relacionados a eventos já foram utilizados para avaliação de memorização de palavras, mostrando lateralização para o LTM esquerdo(24), e de estímulos não verbais, sem lateralização definida ${ }^{(37)}$. Mas os paradigmas em bloco são o principal método utilizado para obter as lateralizações de LTM citadas acima, que variam de acordo com o grau de "verbalizidade" do material memorizado.

Outro aspecto pouco estudado da ativação hipocampal por RMF é a diferença de lateralização em diferentes regiões do hipocampo ou do LTM. A experiência clínica em pacientes com distúrbios de memória após ressecção do LTM anterior sugere que as regiões anteriores sejam cruciais para a memorização de novas informações. No entanto, a maior parte dos estudos funcionais revela ativações principalmente nas regiões posteriores do hipocampo e do $\operatorname{LTM}^{(11,13,15,16,22,26,37,38)}$. Isso pode estar relacionado com artefatos de susceptibilidade presentes nas regiões anteriores ${ }^{(39)}$ ou com a influência de ativações em áreas adjacentes ao LTM posterior, como giro fusiforme e 
outras regiões occipitais, que naturalmente se ativam em tarefas de memorização de estímulos visuais. Etapas de préprocessamento, como suavização e normalização podem fazer as ativações occipitais se expandirem artificialmente para as regiões posteriores do LTM.

Por fim, um dos grandes problemas de estimar índices de lateralização (IL) para ativações de LTM é que ativações nessa região são caracteristicamente fracas, produzindo apenas alguns poucos voxels ativos acima de limiares estatísticos convencionais, tais quais $P=0.05$ ou $P=0.01$. Esses limiares, além do problema de serem arbitrariamente escolhidos, podem levar a situações conflitantes, como lateralizações para um lado usando determinado valor de $P$, e para outro, usando outro valor de $P$. Além disso, ILs podem produzir valores extremados, como +1 ou -1 , apenas pela presença de alguns poucos voxels ativos em um lado e nenhum voxel ativo no outro. Essas situações são encontradas com freqüência em análises do LTM. A maior parte dos estudos de RMF para mapeamento de memória, no entanto, tem seguido a abordagem padrão de arbitrariamente selecionar um limiar estatístico, com valores de $P$ variando na literatura entre 0.00003 e $0.01^{(20-27)}$. Devido ao fato de que a pesquisa com RMF se baseia fundamentalmente nos mapas de ativação (que contêm apenas voxels acima de um determinado limiar estatístico), nenhum estudo de memória utilizou em sua análise voxels fracamente ativados (abaixo do limiar estatístico), os quais ainda podem ser úteis, se considerarmos que o limiar foi escolhido arbitrariamente. Esses voxels podem ser particularmente úteis se o objetivo for apenas calular Ils - e não mapas de ativação - pois eles podem pelo menos indicar o lado de maior ativação (mesmo que não seja possível produzir mapas dessa ativação).

Alguns estudos de RMF têm tentado aplicar limiares mais baixos para incluir na análise esses voxels com menor significância estatística, mas poucos têm explorado o uso de metodologias totalmente independentes de limiar estatístico $^{(40)}$. Isso é parcialmente conseqüência do fato de que os ILs têm sido calculados primariamente para avaliar lateralização de linguagem ${ }^{(21,41-45)}$, que envolve regiões cerebrais muito mais amplas e ativações com uma boa relação sinal/ruído. Devido ao grande número de voxels ativados, a abordagem tradicional de selecionar um limar e simplesmente comparar o número de voxels em cada lado acima do limiar é normalmente suficiente para identificar o lado dominante. O LTM, por outro lado, é pequeno e as ativações de memória dependem de todas as demais variáveis descritas acima. Novos estudos, portanto, que lancem mão de formas alternativas de calcular ILs no LTM, são altamente necessários.

Esses estudos são necessários também para que a RMF de memória possa finalmente ser validada para uso clíni- co. Poucos estudos têm procurado comparar ILs obtidos de pacientes com aqueles obtidos de grupos de sujeitos normais. A função de identificar lateralizações patológicas em pacientes tem sido suprida pelo TAS, mas essa técnica normalmente produz apenas resultados qualitativos, tais como "para a esquerda", "para direita" ou "bilateral". Embora até seja possível calcular ILs a partir do número de objetos lembrados após o efeito anestésico, a falta de um grupo controle no TAS nos impede de saber quão diferente é a lateralização dos pacientes em comparação à população normal. A técnica de RMF, no entanto, nos permite estudar grupos-controle e obter valores de referência para serem usados com pacientes. Utilizando RMF, é possível saber quantos desvios-padrão o IL de determinado paciente está desviado da média da população normal. Um resultado de TAS indicando "lateralização para a esquerda" em um paciente informa muito menos de que um resultado de RMF indicando um "desvio para a esquerda maior do que dois desvios-padrão da população", por exemplo. Como é esperado encontrar dominância cerebral à esquerda, principalmente para linguagem, mas também para memória, é importante que se possa quantificar essa lateralização em comparação ao um grupo controle.

Apesar das dificuldades descritas acima, a RMF combinada com outras técnicas de mapeamento cerebral funcional possui um futuro promissor em um presente no qual já é possível obter ILs confiáveis para a memória - utilizando os equipamentos e técnicas adequadas. Em função de melhores características de sinal e ruído, os aparelhos de ressonância magnética de 3T são claramente superiores aos aparelhos de 1,5T para estudos de memória. Eles não apenas são melhores para mensurar o sinal BOLD (Blood Oxygen Level Dependent) de RMF em regiões vulneráveis a artefatos de susceptibilidade magnética (como as regiões anteriores do LTM), como também apresentam um maior potencial para identificar variações discretas do sinal BOLD. Como a memória é um processo contínuo, que não pode ser voluntariamente interrompido (ao contrário da linguagem e de tarefas motoras), os paradigmas de memória normalmente buscam comparar momentos de "maior atividade de memória" com momentos de "menor atividade de memória". Isso é alcançado através do grau de novidade dos estímulos apresentados. De forma geral, é preciso comparar estímulos que foram vistos apenas uma vez (com alto "grau de novidade") com estímulos repetidos (baixo "grau de novidade"). Essa diferença é provavelmente bem menor do que os usuais 2-5\% encontrados em tarefas motoras, tornando o uso de aparelhos de 3T ainda mais importante. Particularmente, o uso de seqüências espirais de aquisição ${ }^{(39)}$ pode ajudar a minimizar artefatos de susceptibilidade. 
Com a relação à técnica, algumas variáveis podem ser trabalhadas de forma a produzir resultados mais consistentes. Paradigmas em bloco permanecem sendo os ideais para aplicação clínica. Apesar de não ser possível verificar o efeito da memorização bem sucedida com paradigmas em bloco, dados ainda não publicados do nosso laboratório sugerem que, pelo menos em sujeitos normais, as diferença entre estímulos com alto e baixo grau de novidade é aproximadamente a mesma que aquela entre estímulos com alto grau de novidade e que foram memorizados com sucesso, e estímulos de baixo grau de novidade. Em pacientes que possuem um distúrbio de memória pré-operatório importante, e que portanto memorizam poucos estímulos, o uso de apenas estímulos memorizados com sucesso pode produzir melhores resultados do que o uso de todos os estímulos com alto grau de novidade (sejam eles lembrados ou esquecidos). No entanto, se o número de estímulos lembrados for extremamente baixo, pode simplesmente não haver estímulos suficientes para gerar um contraste e conseqüentemente um mapa de ativação. Para solucionar esse problema, é possível que o tamanho do paradigma precise ser ajustado para cada indíviduo, de acordo com a porcentagem de estímulos que aquele indivíduo normalmente consegue memorizar. A aplicação dessa estratégia, no entanto, ainda não foi apresentada na literatura. Portanto, enquanto não estiver melhor esclarecido como pacientes com dificuldade de memorização devem ser estudados, exames funcionais de memória nesses pacientes devem ser conduzidos com cautela. No entanto, a mesma ressalva vale para o TAS, sendo que a RMF nos oferece o potencial de aumentar consideravelmente $\mathrm{o}$ tamanho da sessão de memorização, enquanto que qualquer aumento de duração do TAS pode ser complicado por fatores relacionados à anestesia e ao procedimento.

Com relação aos ILs, é importante que lateralizações de memória obtidas por RMF em pacientes sejam comparadas com uma distribuição de lateralizações obtidas de uma população normal. Naturalmente, a técnica de cálculo de ILs deve ser a mesma para o grupo controle e para os pacientes. De preferência, a técnica utilizada deve produzir resultados robustos (independentes de parâmetros arbitrários, como limiares estatísticos) e deve permitir a comparação entre sujeitos ${ }^{(40)}$. Recentemente, nós propusemos (artigo submetido e em revisão) que esses requisitos podem ser atendidos por uma técnica que utilize todos os voxels positivamente correlacionados com a tarefa (com valores de $T>0$ ) e que leve em consideração a importância (ou significância estatística) de cada voxel. Essa técnica seria mais robusta que a técnica tradicional de simplesmente contar os voxels acima de determinado limiar, pois seria independente de parâmetros arbitrários. Além disso, ela seria mais consistente, pois resultaria em menor varia- bilidade entre sujeitos, permitindo uma comparação mais clara entre pacientes e o grupo controle.

Ainda com relação à técnica, é importante mencionar também que é altamente recomendável que exames clínicos de RMF de memória sejam realizados durante a fase de memorização. A grande maioria dos estudos tem focado na memorização e não no reconhecimento, pois os resultados costumam ser muito mais consistentes durante a memorização do que durante o reconhecimento.

Por fim, é importante levar em consideração o material memorizado. Como existe uma importante influência do tipo de material, é interessante que cada sujeito seja estudado durante a memorização de palavras (com alto grau de verbalizidade), de padrões (com baixo grau), e de imagens com conteúdo (como cenas, que possuem um grau de verbalizidade intermediário). Como cada uma dessas modalidades de estímulo pode produzir diferentes lateralizações, é preciso combiná-las em uma única análise estatística para que se possa obter uma lateralização média ou uma lateralização de memória global, independente de material. Dados ainda não publicados do nosso laboratório sugerem que a análise combinada dessas modalidades permite uma melhor diferenciação entre pacientes e o grupo controle. Isso se deve provavelmente ao efeito combinado do balanceamento de modalidades e do uso de um maior número de estímulos na análise do que é utilizado na análise de cada uma dessas modalidades individualmente.

Para o futuro do mapeamento de memória, espera-se obter um grande refinamento das técnicas atuais. É possível que paradigmas relacionados a eventos, adaptados às necessidades específicas de cada paciente, se tornem a regra. $\mathrm{O}$ aumento da experiência com exames obtidos durante a fase de reconhecimento pode revelar o valor clínico dessa etapa específica do processo de memorização (revelando informações sobre o processo subjacente de consolidação de memória), que por enquanto tem sido utilizado basicamente para categorizar os estímulos apresentados durante a memorização. Mas os maiores avanços deverão vir da combinação da RMF em aparelhos de 3T com estudos estruturais realizados nesses mesmos aparelhos e estudos funcionais realizados por eletrocorticografia (ECoG) e magnetoencefalografia (MEG). Em nosso laboratório, estamos atualmente produzindo os primeiros mapas combinados de RMF com MEG e de RMF com tratografia. Apesar de estarmos testando a combinação de técnicas em estudos de linguagem, a aplicação dessas técnicas combinadas em memória é apenas uma questão de tempo. Além de simplesmente corregistrar os resultados das diferentes ténicas em um mesmo mapa, informações de uma determinada técnica podem ajudar na execução de uma outra técnica. Por exemplo, é possível identificar 
tratos que se originam exclusivamente em determinadas regiões de interesse identificadas previamente por RMF ou por MEG. No futuro, mapas funcionais não incluirão apenas informações de distribuição espacial das áreas identificadas por RMF, mas também informações de distribuição temporal, obtidas através de técnicas de alta resolução temporal, como ECoG e MEG. Finalmente, a combinação de ECoG e RMF nos permitirá identificar a seqüência de ativação das áreas identificadas por RMF. É provável que um dia estejamos analisando "vídeos" de ativação funcional de memória e não apenas "fotos" desse fenômeno.

\section{REFERÊNCIAS}

1. Scoville W, Milner B. Loss of recent memory after bilateral hippocampal lesions. J Neurol Neurosurg Psychiatry 1957; 20(1):11-21.

2. Squire L. Memory and the hippocampus: a synthesis from findings with rats, monkeys, and humans. Psychol Rev 1992; 99(2):195-231.

3. Eichenbaum H. A cortical-hippocampal system for declarative memory. Nat Rev Neurosci 2000; 1(1):41-50.

4. Squire L Zola-Morgan S. The medial temporal lobe memory system. Science 1991; 253(5026):1380-6.

5. Akanuma $\mathrm{N}$ et al. Presurgical assessment of memory-related brain structures: the Wada test and functional neuroimaging. Seizure 2003; $12(6): 346-58$.

6. Wada J, Rasmussen T. Intracarotid injection of sodium amytal for the lateralization of cerebral speech dominance: experimental and clinical observations. J Neurosurgery 1960; 17:266-82.

7. Milner B, Branch C, Rasmussen T. Study of short-term memory after intracarotid injection of sodium amytal. Transactions of the American Neurological Association 1962; 87:224-6.

8. Jack CJ et al. Selective posterior cerebral artery injection of amytal: new method of preoperative memory testing. Mayo Clin Proc 1989; 64(8):965-75.

9. Simkins-Bullock J. Beyond speech lateralization: a review of the variability, reliability, and validity of the intracarotid amobarbital procedure and its nonlanguage uses in epilepsy surgery candidates. Neuropsychol Rev 2000; 10(1):41-74.

10. Dion J et al. Clinical events following neuroangiography: a prospective study. Stroke 1987; 18(6):997-1004.

11. Golby A et al. Material-specific lateralization in the medial temporal lobe and prefrontal cortex during memory encoding. Brain 2001; 124(Pt 9):1841-54.

12. Schacter D, Wagner A. Medial temporal lobe activations in fMRI and PET studies of episodic encoding and retrieval. Hippocampus 1999; 9(1):7-24.

13. Kelley D et al. Hemispheric specialization in human dorsal frontal cortex and medial temporal lobe for verbal and nonverbal memory encoding. Neuron 1998; 20(5):927-36.

14. Wagner D et al. Material-specific lateralization of prefrontal activation during episodic encoding and retrieval. Neuroreport 1998; 9(16):3711-7.

15. Gabrieli J et al. Separate neural bases of two fundamental memory processes in the human medial temporal lobe. Science 1997; 276(5310):264-6.

16. Stern $\mathrm{C}$ et al. The hippocampal formation participates in novel picture encoding: Evidence from functional magnetic resonance imaging. Proc Natl Acad Sci 1996; 93(16):8660-5.

17. Dolan R, Fletcher P. Encoding and retrieval in human medial temporal lobes: An empirical investigation using functional magnetic resonance imaging (fMRI). Hippocampus 1999; 9(1):25-34.
18. Martin A. Automatic activation of the medial temporal lobe during encoding: Lateralized influences of meaning and novelty. Hippocampus 1999; 9(1):62-70.

19. Machado V, Costa J da. Estudo comparativo entre a ressonância magnética funcional e o teste do amobarbital na avaliação da lateralização da memória em pacientes com epilepsia refratária do lobo temporal [dissertação],:Porto Alegre: PUCRS; 2002.

20. Golby A et al. Memory lateralization in medial temporal lobe epilepsy assessed by functional MRI. Epilepsia 2002; 43(8): 855-63.

21. Deblaere $\mathrm{K}$ et al. Developing a comprehensive presurgical functional MRI protocol for patients with intractable temporal lobe epilepsy: a pilot study. Neuroradiology 2002; 44(8):667-73.

22. Detre J et al. Functional MRI lateralization of memory in temporal lobe epilepsy. Neurology 1998; 50(4):926-32.

23. Killgore W et al. Functional MRI and the Wada test provide complementary information for predicting post-operative seizure control. Seizure 1999; 8(8):450-5.

24. Richardson $M$ et al. Preserved verbal memory function in left medial temporal pathology involves reorganisation of function to right medial temporal lobe. NeuroImage 2003; 20(Suppl 1): S112-9.

25. Bellgowan P et al. Side of seizure focus predicts left medial temporal lobe activation during verbal encoding. Neurology 1998; $51(2): 479-84$.

26. Dupont $S$ et al. Episodic memory in left temporal lobe epilepsy: a funcional MRI study. Brain 2000; 123(Pt 8):1722-32.

27. Jokeit H, Okujava M, Woermann F. Memory fMRI lateralizes temporal lobe epilepsy. Neurology 2001; 57(10):1786-93.

28. Tulving $\mathrm{E}$ et al. Hemispheric encoding/retrieval asymmetry in episodic memory: Positron emission tomography findings. Proc Natl Acad Sci 1994; 91:2016-20.

29. Nyberg L et al. General and specific brain regions involved in encoding and retrieval of events: What, where, and when. Proc Natl Acad Sci 1996; 93:11280-5.

30. Habib R, Nyberg L, Tulving E. Hemispheric asymmetries of memory: the HERA model revisited. TRENDS in Cognitive Sciences 2003; 7(6):241-5.

31. Frisk V, Milner B. The role of the left hippocampal region in the acquisition and retention of story content. Neuropsychologia 1990; 28(4):349-59.

32. Ivnik R, Sharbrough F, Laws EJ. Effects of anterior temporal lobectomy on cognitive function. J Clin Psychol 1987; 43(1): 128-37.

33. Smith M, Milner B. The role of the right hippocampus in the recall of spatial location. Neuropsychologia 1981; 19(6):781-93.

34. Spiers $\mathrm{H}$ et al. Unilateral temporal lobectomy patients show lateralized topographical and episodic memory deficits in a virtual town. Brain 2001; 124(Pt12):2476-89.

35. McDermott $\mathrm{K}$ et al. Set- and Code-specific activation in the frontal cortex: An fMRI study of encoding and retrieval of faces and words. J Cognitive Neuroscience 1999; 11(6):631-40.

36. Martin A, Wiggs C, Weisberg J. Modulation of human medial temporal lobe activity by form, meaning, and experience. Hippocampus 1997; 7:587-93.

37. Fernandez $G$ et al. Successful verbal encoding into episodic memory engages the posterior hippocampus: a parametrically analyzed functional magnetic resonance imaging study. J Neurosci 1998; 18:1841-7.

38. Kirchhoff B et al. Prefrontal-temporal circuitry for episodic encoding and subsequent memory. J Neurosci 2000; $20(16): 6173-80$.

39. Glover G, Law C. Spiral-in/out BOLD fMRI for increased SNR and reduced susceptibility artifact. Magn Reson Med 2001; 46(3):515-22. 


\section{Branco D, Costa JC}

40. Nagata $S$ et al. Method for Quantitatively evaluating the lateralization of linguistic function using functional MR Imaging. Am J Neuroradiol 2001; 22(5):985-91.

41. Adcock J et al. Quantitative fMRI assessment of the differences in lateralization of language-related brain activation in patients with temporal lobe epilepsy. Neuroimage 2003; 18(2):423-38.

42. Binder $\mathrm{J}$ et al. Determination of language dominance using functional MRI: A comparison with the Wada test. Neurology 1996; 46(4):978-84.

43. Springer J et al. Language dominance in neurologically normal and epilepsy subjects: a functional MRI study. Brain 1999; $122(\mathrm{Pt}$ 11):2033-46.
44. Rutten $\mathrm{G}$ et al. FMRI-determined language lateralization in patients with unilateral or mixed language dominance according to the Wada test. Neuroimage 2002; 17(1):447-60.

45. Sabbah P et al. Functional MR imaging in assessment of language dominance in epileptic patients. Neuroimage 2003; 18(2):460-7.

\section{Endereço para correspondência:}

Daniel Branco

Department of Neurosurgery, Brigham and Women's Hospital, Harvard Medical School - 75 Francis Street, Room CA138-B

Boston, MA 02115

E-mail:branco@bwh.harvard.edu 